\title{
A PROPERTY OF THE RAYLEIGH FUNCTION
}

\section{F. T. HOWARD}

1. Introduction. Let $J_{\nu}(z)$ denote the Bessel function of the first kind. Kishore [3], [4], has defined the Rayleigh function of order $2 n$ by means of

$$
\sigma_{2 n}(\nu)=\sum_{m=1}^{\infty}\left(j_{\nu, m}\right)^{-2 n} \quad(n=1,2, \cdots)
$$

where the $j_{v, m}$ are the zeros of $z^{-\nu} J_{\nu}(z),\left|R\left(j_{v, m}\right)\right| \leqq\left|R\left(j_{\nu, m+1}\right)\right|$. The Rayleigh function is a rational function of $\nu$ and the following recurrence formula has been developed for it [3]:

$$
(\nu+n) \sigma_{2 n}(\nu)=\sum_{k=1}^{n-1} \sigma_{2 k}(\nu) \sigma_{2 n-2 k}(\nu) .
$$

It would be of some interest to know exactly which primes divide the numerator or denominator of $\sigma_{2 n}(\nu)$, if $\nu$ is rational and $\sigma_{2 n}(\nu)$ is reduced to its lowest terms. Restricting ourselves to the prime 2, and using induction on (1.1), we shall find in this paper the exact power of 2 dividing $\sigma_{2 n}(\nu)$ when $\nu$ is a rational number $a / b, a$ odd and $b$ even. This will extend the results of [2] where the same problem was solved for $\nu$ one half an odd integer. In this paper we shall also prove some congruences $(\bmod 2),(\bmod 4)$, and $(\bmod 8)$. We note that this type of problem has been considered for other sequences of rational numbers. For the well-known Bernoulli numbers $B_{2 n}$, for example, it has been proved [1] that

$$
\begin{aligned}
2 B_{2 n} & \equiv 1(\bmod 4)(n>1) \\
& \equiv 1+4 n(\bmod 8)(n>1) \\
& \equiv 1+4 n(\bmod 16)(n>2) .
\end{aligned}
$$

Throughout this paper we shall assume $a$ is an odd integer, $b$ is an even integer, $b=(2 k+1) 2^{t}, t>0$, and $a / b$ has been reduced to its lowest terms.

\section{The power of 2 dividing the Rayleigh function.}

Definition 2.1. Define $\theta_{2 n}(\nu)$ as the exponent of the highest power of 2 dividing the denominator of $\sigma_{2 n}(\nu)$. If $\theta_{2 n}(\nu)$ is negative, it is under1969.

Presented to the Society, November 21, 1969; received by the editors March 26, 
stood that $-\theta_{2 n}(\nu)$ is the exponent of the highest power of 2 dividing the numerator of $\sigma_{2 n}(\nu)$.

Since we shall be proving our main results by using induction on (1.1), it is useful to list here the first three values of $\sigma_{2 n}(a / b)$.

$$
\begin{aligned}
& \sigma_{2}(a / b)=b / 4(a+b), \\
& \sigma_{4}(a / b)=b^{3} / 2^{4}(a+b)^{2}(a+2 b), \\
& \sigma_{6}(a / b)=b^{5} / 2^{5}(a+b)^{3}(a+2 b)(a+3 b) .
\end{aligned}
$$

Theorem 2.1. Suppose $a$ is odd, $b=(2 k+1) 2^{t}, t>0$, and

$$
2 n=2^{s_{1}}+\cdots+2^{s_{m}}, \quad s_{1}>\cdots>s_{m}>0 .
$$

Then

$$
\theta_{2 n}(a / b)=2 n+1+(1-2 n) t-m .
$$

Proof. The theorem is true for $n=1,2,3$. Assume it is true for $1, \cdots, n-1$. Let $x=2 n+1+(1-2 n) t-m$. From (1.1) we have

$$
2^{x}(a+b n) \sigma_{2 n}(a / b)=2^{x} b \sum_{k=1}^{[n / 2]} \alpha_{k} \sigma_{2 k}(a / b) \sigma_{2 n-2 k}(a / b)
$$

where $\alpha_{k}=1$ if $n=2 k, \alpha_{k}=2$ if $n \neq 2 k$. Suppose $m>1$. For a fixed $k$ write $2 k=2^{r_{1}}+\cdots+2^{r_{u}}$ and $2 n-2 k=2^{q_{1}}+\cdots+2^{q_{w}}$. By our induction hypothesis, the exponent of the highest power of 2 dividing the numerator of $2^{x} b \alpha_{k} \sigma_{2 k}(a / b) \sigma_{2 n-2 k}(a / b)$ is $u+w-m$ (or $m-1$ if $n=2 k)$. Hence all terms on the right side of (2.1) are congruent to $0(\bmod 2)$ except those for which

$$
\begin{gathered}
2 k=2^{r_{1}}+\cdots+2^{r_{u}}, \quad 2 n-2 k=2^{q_{1}}+\cdots+2^{q_{w}}, \\
u+w=m .
\end{gathered}
$$

There are $C_{m, 1}$ such terms with $u=1, w=m-1 ; C_{m, 2}$ terms with $u=2$, $w=m-2$; etc. It is easily seen there is a total of $2^{m-1}-1$ terms satisfying conditions (2.2). Hence there are an odd number of terms on the right side of $(2.1)$ congruent to $1(\bmod 2)$. Thus we have

$$
2^{x} \sigma_{2 n}(a / b) \equiv 1(\bmod 2) .
$$

If $m=1$, we need only consider the term on the right of (2.1) such that $2 n-2 k=2 k=n$ and the theorem follows.

3. Congruences $(\bmod 4)$ and $(\bmod 8)$. If $2 n=2^{u}, u>1$, it is easy to raise the modulus in Theorem 2.1 to 4 and to 8 . Again we assume that $b=(2 k+1) 2^{t}, t>0$. 
THEOREM 3.1. If $2 n=2^{u}$, we have

$$
\begin{aligned}
2^{\theta_{2 n}(a / b)} \sigma_{2 n}(a / b) & \equiv(2 k+1) / a(\bmod 4) & & (u>1), \\
& \equiv 5(2 k+1) / a(\bmod 8) & & (u>2) .
\end{aligned}
$$

Proof. To prove the congruence $(\bmod 8)$, we use Theorem 2.1 and formula (2.1). Note that $n=2^{u-1}, n / 2=2^{u-2}, 3 n / 2=2^{u-1}+2^{u-2}$. We have

$$
\begin{aligned}
2^{\theta_{2 n}(a / b)} \sigma_{2 n}(a / b) \equiv & \left(\frac{2 k+1}{a}\right)\left(2^{\theta_{n}(a / b)} \sigma_{n}(a / b)\right)^{2} \\
& +4\left(2^{\theta_{3 n / 2}(a / b)} \sigma_{3 n / 2}(a / b) \cdot 2^{\theta_{n / 2}(a / b)} \sigma_{n / 2}(a / b)\right) \\
\equiv & 5(2 k+1) / a(\bmod 8) .
\end{aligned}
$$

The next two theorems follow in much the same way from Theorem 2.1 and formula (2.1).

TheOREM 3.2. If $2 n=2^{u}+2^{v}$, then

$$
\begin{aligned}
2^{\theta_{2 n}(a / b)} \sigma_{2 n}(a / b) & \equiv(2 k+1) / a(\bmod 4) & & (u=v+1, v \geqq 1), \\
& \equiv 3(2 k+1) / a(\bmod 4) & & (u-v>1, v \geqq 1) .
\end{aligned}
$$

Theorem 3.3. If $2 n=2^{u}+2^{v}+2^{w}$, then

$$
\begin{aligned}
& 2^{\theta+(a / b)} \sigma_{2 n}(a / b) \\
& \equiv(2 k+1) / a(\bmod 4) \quad(u=w+2, v=w+1, w \geqq 1), \\
& \equiv(2 k+1) / a(\bmod 4) \quad(u-v>1, v-w>1, w \geqq 1), \\
& \equiv 3(2 k+1) / a(\bmod 4) \quad(u>3, v=2, w=1) \text {, } \\
& \equiv 3(2 k+1) / a(\bmod 4) \quad(u-v=1, v-w>1, w \geqq 1) .
\end{aligned}
$$

In order to find more general congruences $(\bmod 4)$ we need the following definition.

Definition 3.1. Let $2 n=2^{s_{1}}+\cdots+2^{s_{m}}, s_{1}>\cdots>s_{m}>0$. Define $\psi(2 n)$ as the number of positive integers $2 k, 2 k \leqq n$, such that

$$
\begin{gathered}
2 k=2^{r_{1}}+\cdots+2^{r_{u}}, \quad r_{1}>\cdots>r_{u}>0, \\
2 n-2 k=2^{q_{1}}+\cdots+2^{q_{w}}, \quad q_{1}>\cdots>q_{w}>0, \\
u+w=m+1 .
\end{gathered}
$$

The following lemma is important in the proofs of Theorems 3.4-3.7.

Lemma 3.1. Let $2 n=2^{s_{1}}+\cdots+2^{s_{m}}, s_{1}>\cdots>s_{m}>0$. Suppose in this expansion there are $h_{1}$ "single" terms $2^{s_{i}}$ such that 


$$
s_{i-1}-1>s_{i}>s_{i+1}+1,
$$

$h_{2}$ "doubles" $2^{s_{i}}, 2^{s_{i+1}}$ such that

$$
s_{i-1}-1>s_{i}=s_{i+1}+1>s_{i+2}+2,
$$

$h_{3}$ "triples," etc. Then

$$
\begin{aligned}
\psi(2 n) & =2^{m-2} \sum_{i=1}^{m} h_{i} \quad \text { if } s_{m}>1 \\
& =2^{m-2}\left(\sum_{i=1}^{m} h_{i}-1\right) \quad \text { if } s_{m}=1 .
\end{aligned}
$$

We shall continue to use the terms "single," "double," and "triple" in the sense of Lemma 3.1.

THEOREM 3.4. Let $2 n=2^{s_{1}}+\cdots+2^{s_{m}}, s_{1}>\cdots>s_{m} \geqq 1$. If each term of this expansion is "single" in the sense of Lemma $3.1\left(s_{i}-s_{i+1}>1\right.$ for $i=1, \cdots, m-1)$, then

$$
2^{\theta_{2 n}(a / b)} \sigma_{2 n}(a / b) \equiv(-1)^{m+1}(2 k+1) / a(\bmod 4) .
$$

Proof. The proof is by induction on $m$. The theorem is true for $m=1,2,3$. Assume it is true for $1,2, \cdots, m-1$, and let $2 n=2^{s_{1}}$ $+\cdots+2^{s_{m}}$. By Lemma 3.1, we know $\psi(2 n) \equiv 0(\bmod 2)$. Hence in formula (2.1) we need only consider terms on the right side satisfying (2.2). There are $2^{m-1}-1$ such terms. Let $g(2 n)$ be the number of these terms congruent to $-1(\bmod 4)$. Then, if $s_{m}>1$,

$$
2^{\theta 2_{n}(a / b)} \sigma_{2 n}(a / b) \equiv \frac{(2 k+1)}{a}\left[2^{m-1}-1-2 g(2 n)\right](\bmod 4) \text {. }
$$

If $m$ is even, then, by our induction hypothesis, $g(2 n)=0$. If $m$ is odd, then $g(2 n)=2^{m-1}-1$, and the theorem follows for $s_{m}>1$. If $s_{m}=1$ the proof is the same, except that in formula (3.1) $(2 k+1) / a$ must be replaced by $(2 k+1) /\left(a+2^{t}\right)$, and it must be remembered that $2^{\theta_{2}(a / b)} \sigma_{2}(a / b) \equiv(2 k+1) /\left(a+2^{t}\right)(\bmod 4)$.

The proofs of Theorems 3.5-3.7 are similar.

THEOREM 3.5. Let $2 n=2^{s_{1}}+\cdots+2^{s_{m}}, s_{1}>\cdots>s_{m} \geqq 1$. Suppose in this expansion there are $m-2$ "singles" and one "double." Then

$$
2^{\theta_{2 n}(a / b)} \sigma_{2 n}(a / b) \equiv(-1)^{m}(2 k+1) / a(\bmod 4) .
$$

THEOREM 3.6. Let $2 n=2^{s_{1}}+\cdots+2^{s_{m}}, s_{1}>\cdots>s_{m} \geqq 1$. If in this expansion there are one "triple" and $m-3$ "singles," then

$$
2^{\theta_{2 n}(a / b)} \sigma_{2 n}(a / b) \equiv(-1)^{m+1}(2 k+1) / a(\bmod 4) .
$$


TheOREM 3.7. Let $2 n=2^{s_{1}}+\cdots+2^{s_{m}}, s_{1}>\cdots>s_{m} \geqq 1$. If in this expansion there are $h$ "doubles" ( $h$ pairs satisfying the definition in Lemma 3.1), and $m-2 h$ "singles," then

$$
2^{\theta_{2 n}(a / b)} \sigma_{2 n}(a / b) \equiv(-1)^{h+m+1}(2 k+1) / a(\bmod 4) .
$$

4. A conjecture. The results of Theorems 3.4-3.7 lead us to the following conjecture.

CONJECTURE. Let $2 n=2 s_{1}+\cdots+2^{s_{m}}, s_{1}>\cdots>s_{m} \geqq 1$. If in this expansion there are $h_{1}$ "singles," $h_{2}$ "doubles," $h_{3}$ "triples," etc., then letting $\sum_{1}^{m} h_{i}=h$, we have

$$
2^{\theta_{2 n}(a / b)} \sigma_{2 n}(a / b) \equiv(-1)^{h+1}(2 k+1) / a(\bmod 4) .
$$

\section{REFERENCES}

1. L. Carlitz, A property of the Bernoulli numbers, Amer. Math. Monthly 67 (1960), 1011-1021.

2. F. T. Howard, Generalized van der Pol numbers, Math. Nachr. (to appear).

3. N. Kishore, The Rayleigh function, Proc. Amer. Math. Soc. 14 (1963), 527-533. MR 27 \#1633.

4. - The Rayleigh polynomial, Proc. Amer. Math. Soc. 15 (1964), 911-917. MR $29 \# 6079$.

WAKE Forest UNIVERSITY 